\title{
Index
}

Agreement on Cooperation in Research,

Conservation and Management of Marine

Mammals in the North Atlantic $\quad 337-338$

Agreement on Cooperation on Aeronautical and Maritime Search and Rescue in the

Arctic 72 n. 26, 192 n. $115^{-116,} 196$ n. 143, $146,25^{8}$

Alaska $\quad 72$ n. 27,77 n. 43, 84 n. 65 ,

94 n. $87,107,121$ n. $6,125^{-127}$ n. 22 , 129, 145 n. 66-68, 153 n. 90, 201, 202 n. 8, 203-221 n. 95, 223 n. 98, 103, 224 n. 106, 108,225 n. 109, 227 n. 117, 228 n. 123, 230, 231 n. 130-131, 133, 232-234 n. 138, 255 n. $67,70,266$ n. 107, 279, 306 n. 32, 316 n. 82

Antarctic 108, 155, 172 n. 4, 181 n. 54, 257, 258 n. 80,261 n. $91,286,326$ n. 123

Antarctic Treaty $108,155,286$

Arctic 1-7, 9, 11-43, 42 n. 14, 46-50, 52-63, $65^{-67}, 68$ n. 10, 71-73, 75-83, 84 n. 65 , 85,86 n. 69,87 n. 70, 88-89, 91-100, 102, 104-211, 212 n. 53, 214, 216 n. 72, 217-218, 220, 222, 223 n. $98,224,226$ n. 115 , 227, 229, 231, 232, 233 n. 136, 234, 237, 239-301, 303 n. 16,305 n. 32, 309 n. 47 , 310 n. 53 , 315 n. 75, 316-317, 318 n. 89 , 321-322, 324, 325-328, 336 n. 187, 337 n. 193, 339-350, 353-359

Arctic Athabaskan Council (AAC) 75 n. 36 , 125, 208 n. 29, 228 n. 123

Arctic Circle $\quad 20,49,82-83,104,106$, 112, 172 n. 1,187 n. $89,201,206,210,275$ n. 7

\section{Arctic Council}

Conservation of Arctic Flora and Fauna Working Group (CAFF) 16, 146, 247 n. 35

Emergency Prevention, Preparedness and Response (EPPR) Working Group 270

Iqaluit Declaration $\quad 40$ n. 1, 136 n. 42, 145

Ministerial (Meetings) 27 n. 83, 40, 48 n. 34,49 n. $36,50,54-56$ n. 61 , 142 n. $57,165,170,195$ n. 136, 200, 209, 244, 249

Observership $\quad 40,60,170$

Ottawa Declaration 122, 244 n. 16
Protection of the Arctic Marine Environment (PAME) Working Group 144, 161, 248, 275 n. 7

Permanent Participants $\quad 93,132$, 141-143 n. 61, 145, 165-166, 208

Secretariat 44 n. 21, 45-46 n. 27, 51, 56,8 o n. 52, 141 n. $54,145,170$ n. 148 , 276 n. $15,293,294$ n. 128

Senior Arctic Officials (SAOs) $\quad 141,142$ n. 58,163 n. 128,165 n. 134

Sustainable Development Working Group (SDWG) 144

Arctic Economic Council (AEC) 143

Arctic Environmental Protection Strategy

(AEPS) $\quad 40,270$

Arctic Five

Oslo Declaration $\quad 280,292$

“ $5+5$ " 2 n. 8, 280, 368

Arctic Institute 27 n. 81, 28, 29 n. 91, 83 n. 62,97 n. 1, 105 n. 36, 109 n. 50, 53,110 n. 57,111 n. $60,64,113$ n. 71, 118, 202 n. 10, 208 n. 32, 209 n. 35, 224 n. 107

Arctic Marine Shipping Assessment (AMSA) 143, 147 n. 73, 148 n. 75, 149 n. 80,162 n. 124,164 n. 131,168 n. 142 , 257 n. 77

Arctic Marine Shipping Plan (AMSP) 161 Arctic Ocean

Central Arctic Ocean $\quad 2,6-7,16,33$, 152, 160, 274-277, 279-280 n. 39, 282-283, 285-288, 290-295

Arctic Waters Pollution Prevention Act

(AWPPA) 129, 253 n. 6o

Atlantic Ocean 14, 15 n. 18, 27 n. 81, 275 n. 7

Australia 1 n. 1, 274 n. 1, 278 n. 25, 326 n. 123

Baltic Sea 14 n. 16, 21, 30-31 n. 96, 41 n. 9, $44,46,48$ n. $3^{2}, 5^{1}$ n. $42,5^{2}$ n. $46,53^{-} 5^{6}$ n. 61,58 n. 70,207 n. 27

Banana Hole $\quad 276$

Barents Euro-Arctic Council (BEAC) 4, 16, $28,41-44,46-50,52,54-55,57,60,62$, 207 n. 27,242 n. 9

Barents Regional Council (BRC) 46, 48 


\section{Barents Sea}

Barents $2020 \quad 189,193,356$

Loophole 276, 323

Russian-Norwegian Treaty on Maritime

Delimitation and Cooperation in

the Barents Sea and the Arctic

Ocean 48

Baselines 20, 131, 252 n. 53, 253

Beaufort Sea $123,129,138,158$ n. 108

Belarus 54

Benelux 42

Bering Strait 157,180 n. 46, 201 n. 4, 247

Black Sea $\quad 43,5^{1}$ n. 41

Canada

Arctic Waters Pollution Prevention Act (AWPPA) 129, 253

Berger Inquiry 130

Canada-EU relationship 120

Canada-Greenland Joint Commission on Beluga and Narwhal 336 n. 184

Canadian High Arctic Research Station (CHARS) 140

Indigenous peoples $5-7,15$ n. 17,18 n. 42 , 20, 37 n. 126, 38, 48-49, 66, 72, 73, 74, 75-81, 85-86, 88, 91, 93, 94 n. 85, 96, $98,115^{-116}, 121-126,128,130,134$ n. 38 , 135, 141, 145, 146-149, 152, 162-164 n. 130, 166-168, 203, 205, 208 n. 29, 209-211, 214, 222 n. 98,223 n. $98,225,228$, 229 n. 125, 232, 297-299, 303 n. 18 , 304-306 n. 35, 309, 314-317, 322, 325, $331,340,361-363$

NORDREG 139 n. 50, 255, 260

Canadian Forces Northern Area 133 n. 36

Central Arctic Ocean $\quad 2,6-7,16,33,152$, 16o, 274-277, 279-28o n. 39, 282, 283, 285-288, 290-295

Central European Free Trade Agreement (CEFTA) 44

Centre for Strategic and International Studies (CSIS) 174, 175 n. 16

Cetaceans 277 n. 23, 281 n. 42, 299-300, $316-317,319,321,324-325,334-335$

China 2 n. 8, 15, 22, 34, 59 n. 73, 61, 65, $96,160,172,183$ n. 68,202 n. 8 , 208 n. 30, 224, 244 n. 17, 280-281, 292-293
Circumpolar Arctic $\quad 4,11$ n. 1, 13, 19, 26-28, $39,117,118$ n. 86, 120, 171

Climate Change $2-3,6,13,17-18,23$, $27-28,37,40,47,49,57-59,69$, $80-81,89-90$ n. 76,103 n. $22,120,124$, 132-134, 138, 140, 142, 145, 147-149, 151-152, 154, 157-159, 161, 169, 171, 178-179, 242, 246-247 n. 35, 261, 271 n. 123, 272, 275, 277, 285, 293, 295, 345-348 ก. 28,353

Coastal state $121,138,144,155,251^{-252}$, 26o, 262 n. 93, 264-266 n. 107

Cold War 41, 43 n. 15, 99, 121-122, 127, 132, $174,191,198$

Commission on the Limits of the Continental Shelf (CLCS) 82 n. 59, 155

Communism 43

Conservation of Arctic Flora and Fauna (CAFF) Working Group 1 n. 5, 16, 146, 275 n. 8

Continental Shelf 82,84 n. 64, 89, 90, $136,138,149,154-156$ n. 100, 183,188 , 190 n. 101, 196-197 n. 147, 344 n. 11, 354 ก. $47,357,35^{8}$

Convention on Biological Diversity (CBD) 3,277

Convention on International Trade in Endangered Species of Wild Fauna and Flora (CITES) 168, 321, 322 n. 111, 325, 335 n. 178

Cooperation

Sub-regional $\quad 41-46$ n. 26, 6o-62, 207

Council of Baltic Sea States (CBSs) 41, 207 n. 27,234 n. 138

Crustacean 277

Customary law 249 n. 40

Czech Republic $\quad 42,44$

Denmark

Greenland $\quad 3-5,14,15$ n. 18-19, 29, 40-41, $45,53,56-62,65-71,73,76,77$ n. 43 , 79 n. 50, 8o-88 n. 72, 91-96, 107, 123, 125, 128 n. 24, 131, 161 n. 121, 166 n. 137, 171, 172 n. 1, 3, 173, 174, 177, 189 n. 98 , 196, 200, 206-207, 208 n. 29, 31, 209, 221, 224, 226-229, 231, 233, 241, 246 n. 30 , 247, 252, 255, 275, 277 n. 17, 279, 281, 282, 286 n. 77,293 n. 122, 297 n. 3, 299, 
303, 306 n. 32, 311-312, 315-316, 317 n. 88,319 n. $94,322,326$ n. 125 , 327 n. $133,328,330-333,335^{-3} 8$, $340-341,345$ n. $14,356,362$

Donut Hole $\quad 276,279,287$ n. 84

\section{Ecosystem}

Ecosystem approach 344

Ecuador 281

Emergency Prevention, Preparedness and Response (EPPR) Working Group 49 n. 37,270

\section{Energy}

Energy Charter Treaty $\quad 189$

Oil \& gas $\quad 72,82-83,89-91$

Renewables 17,117

Enforcement $\quad 150,218,241,25^{-253}, 259,264$, 266, 269, 271, 357 n. 6o, 359

European Economic Area (EEA)

Agreement on the EEA 101, 106

European Economic Community (EEC) 66, $68,70,319$

European Committee for Standardization (CEN) $356,35^{8}$

European Regional Development Fund (ERDF) 113

European Union (EU) 2012 Arctic Joint Communication 347, 349 2016 Joint Communication $\quad 20,23,25-30$, $35,37-39,342,347$

APPLICATE 349

Arctic Policy $3-5,9,11-13$ n. 8, 20, 21 n. $56,22,23-36,37$ n. 128,39 , 46 n. 26,57 n. $62,59,81,106-108,113$, 115 n. $81,116-117,120,135,137,147$, 153-157, 171-172, 175-179, 181-182, 185, 189-191, 194, 196, 198-203 n. 11, 207, 208 n. 29, 239, 240 n. 3, 241-242, 244-246, 256, 260, 264, 270-271, 274, 276 n. $15,284-285,287,293$ n. 125 , 295-296 n. 1, 318, 324, 326 n. 122, 340 n. $202,342-349,360-361,363-366$, 368

Brussels 3 n. 14, 12 n. 4, 28, 38, 41, 44, 46, 51, 59 n. 71, 61-62, 103-104, 107-109, 110 n. $57,116-117,228$ n. 121,276 n. 15 , 284,285 n. 72,286 n. 73,289 n. 93 ,
290 n. 102,346 n. $18-20,347,348$ n. 26 , $35^{\circ}$ n. $35,35^{6}$ n. $55,35^{8}$ n. 61

Common Fisheries Policy (CFP) 16, 243, 288, 338 n. 197

Council of the European Union 12, 13 n. $7,25,109,115$ n. $80,167,178$ n. 37 , 180 n. 51, 181 n. 57,189 n. 99, 227, 309, 346 n. 18-20

Court of Justice of the European Union (CJEU) 78-79, 93, 164, 225, 283, 298, $300 \mathrm{n} .5$

Directives 91 n. $81,304,343,348$

$1994 / 22 /$ EC $\quad 89$

$2001 / 42 /$ EC 91

$2008 / 56 /$ EC 1 n. 2,343 n. 3

2011/92/EU 20 n. 54, 91

2013/30/EU 7, 33 n. 104, 89-9o, 265 n. 103,342 n. ${ }^{* *}, 351,354,356$

$2014 / 5^{2} / \mathrm{EU} \quad 91$

2014/137/EU 14 n. 15, 68 n. 10, 69, 70 n. 14,241 n. 7,322 n. 109

EURATOM Treaty 71

EU Maritime Safety Agency (EMSA)

270

European Neighbourhood Policy 80 European Parliament 11 n. 1, 25, 27, 29, 35,36 n. 121,41 n. 4 , 45 n. 21,55 n. 57 , 62 n. 8o, 77, 89, 91-92, 105 n. 36, 108, 109, 110 n. 55, 123, 130 n. 29, 153, 155 , 156 n. 101,16 o n. $119,163,165,166,169,177$, 178 n. 36,220 n. $91,223,227$ n. 119, 229 n. $125^{-126,230}$ n. 129,242 n. 9, 245-246 n. $28,260-263$ n. $95,286-287,290$ n. 98 , 296 n. 2, 297 n. 3, 298 n. 4, 304, 307, 309, 313 n. 69,315 n. 78-79, 317 n. 86, 319, 320 n. 100,329 n. 144, 339 n. 200, 342 n. 2, 343 n. 4, 347 nn. 21, 23, 348-349

Habitats Directive $\quad 321-323$

INTAROS 348

INTERACT 96,348

Kolarctic 15, 48 n. 32

Marine Strategy Framework 1, 242 n. 10, $343,346,35^{1}$

Northern Dimension (ND) 4, 15, 41-42, $48,51-57,62,70$ n. 14,79 n. 5 o, 84 n. 64 , 132 n. 35, 201 n. 5, 207 n. 27

ND Partnership on Transport and

Logistics (NDPTL) 51, 55 
European Union (EU) (cont.)

Northern Dimension Environmental Partnership (NDEP) 51, 54

PHARE programme 44, 45 n. 21

Raw Materials Initiative 15,21

Registration, Evaluation, Authorisation and Restriction of Chemicals (REACH) 18 n. 39

Relationship with Canada $\quad$ 119-120, 170

Treaty of the European Union (TEU) $68,77-79$

Treaty on the Functioning of the European Union (TFEU) 14 n. 10, 68, $70,112,224$ n. $107,243,253$ n. 6 o, $282-283$ n. 56 , 309-310, 342-354, 356-359

Exclusive Economic Zone (EEZ) 70, 105, 130, 139, 180, 251, 344 n. 11

Faroe Islands 4,15 n. 19, 40, 45, 56, 67, 94, 206-208 n. 29, 280, 282, 338

Finland $2,11,15$ n. 17, 19-22 n. 64, 29 n. 88, 40, 45-48 n. 32, 76, 99-100, 104, 107, 118,172 n. $3,176,181,195,201,206-208$, 228 n. 123, 232, 239 n. 1, 241, 292, 315, 316 n. 8 o, 319 n. 94, 353

Fisheries

Agreement on Port State Measures $\quad 278$ Compliance Agreement $\quad 278$

Ecosystem approach 344

High Seas $2,7,33,90,160,183,214,251$, $25^{8}$ n. 8 o, 274 n. $5,275,276,277-278$, 280-281, 287-288, 292, 294

IUU Fishing $\quad 278,290-291,295$ IPOA-IUU $\quad 278$

Management $2-3,7,16,19,21,34$, $42-43,48-49,53,58,71,73,75,82,85$, 92, 94 n. $87,105,139-140,146,160,163$, 169,205 n. $16,19,213-216,218$ n. 81 , 219-220, 229, 232, 243, 250, 261, 267, 275-278, 28o, 286 n. 77, 287-292, 294-295, 305 n. 27, 30, 325, 326 n. 121, $327-328,334$ n. $175,337-338,340$, $342,347,352,355,356$ n. 55,357 n. 6 o, 358 n. 62

Maximum sustainable yield (MSY) $\quad 289$ Precautionary approach $15^{2}$

Flag state $\quad 18,250-251,256,264-265,268$, 288, 295
Food and Agriculture Organization (FAO) 276 n. $15,278,281,292,294$

Agreement on Port State Measures $\quad 278$ Compliance Agreement $\quad 278$ IPOA-IUU 278

France $45,208,243$ n. 12, 318 n. 92, 366-377

Galway Statement 14, 159, 160 n. 116

General Agreement on Tariffs \& Trade (GATT) 166 n. 137, 224-226

Germany 41 n. $9,45,126$ n. 19, 156, 243 n. 12, 283,320

Greenland

Canada-Greenland Joint Commission on Beluga and Narwhal 336 n. 184

Naalakkersuisut $5^{\circ}$ n. 41, 58,68,83 n. 63 , 28 o n. 39,290 n. 98

Peoples 73

Act No 473 on Greenland

Self-Government $\quad 67,84$

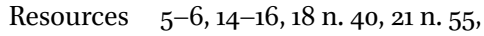
$24,32,34,47,58-60,65,69,71$, $73-75$ n. 36,79 n. $50,80,82-85,87-92$, 95 n. 89-90, 96, 105, 111-112, 120, 122, 124-125, 128-130, 141, 143, 158, 16o, 163, 165 n. $134,172,173,175,176-180,182-184$, 186-191, 193, 196, 198-200, 203 n. 11, 207, 210,213 n. 60,214 n. 66,215 n. 68 , 231-232, 240, 243, 246, 251 n. 48, 255, 256 n. 74, 259 n. 83-84, 269, 274-277, 278 n. 25,279 n. 34,28 n. 39,283 , 289-292 n. 116, 295-296, 305 n. 27, 317 n. $88,339,343$ n. 3, 347-349, 352, 358 n. $61,362-363$

Bureau of Minerals and Petroleum 86-88

Mineral Resources Act $\quad 84-85,88$

Gwich'in Council International (GWI) 125, 145 n. 67,205 n. 19,208

Hans Island $\quad 133,138,154,165$ n. 134 High North $5,7,41,54-55,97,104-108,113$, 114 n. $72,186-187,189,193,197,261$ n. 91 , 296, 298-299, 316, 325, 337-340, 348

High Seas

Fisheries $\quad 2-4,6-7,13,15^{-16,27}, 30$, 32 n. 101, 34, 38 n. 131, 49 n. 35, 58, 59 n. 71, 69, 70, 94, 97 n. 3, 100-102 n. 20, 105 n. 36,109 n. 50, 110, $113,124,141$ n. 54 , 
$15^{2}, 160,168,215^{-216,} 229$ n. 125, 243, 274-295, 302 n. 12, 316 n. 82, 320, 337, 338 n. 197

Hoyvik Agreement 57 n. 62

Human rights $71-72$ n. 29,75 n. 34,76 , 77-78, 93, 94 n. $87,115,163,203$ n. 12, 222, 226, 228, 233, 297, 309 n. 48, 314-317 n. 86

Hungary 42, 44

Ice $1-2$ n. 6,17 n. $35,18,27,5^{8}$ n. $67,65,83$, $120,130,132,138,140,158,172,175$ n. 16 , 179-18o, 185, 187, 196, 198, 202 n. 8, 204 n. 12,205 n. 15,216 n. 72, 246-248, $254,256,265,268,274-275$ n. 6,282 , 296, 305 n. 28,310 n. $5^{1-52,} 3^{22}$ n. 110 , 327 n. 131, 337, 363, 367-368

International Council for Exploration of the Sea (ICES) 294

Iceland $2,4,15,19,29,40-41,45,50,51$ n. 41, $53-54,56,57$ n. $62,59-60,83,94$, 99-100, 102, 16o, 161 n. 121, 164, 172, 176, 195, 241, 259 n. 83, 265-266, 277 n. 17, 19, 280-282, 288, 292, 322, 326 n. 122, 338

Illegal, unregulated and unreported fishing (IUU) 1, 231, 278, 290-291

Ilulissat Declaration 3 n. 17, 136, 250 n. 42, 275 n. 11,286 n. 77

Indigenous peoples

See AAC, Canada, Greenland, GWI, ICC, ILO, UNDRIP

Inuit $7,18,20,27,33,72,75^{-76},=85$, $94-95,122,124-128$ n. $25,133,=135^{-136}$ n. $43,138,139$ n. $50,=141$ n. 54,143 , 147 n. $72,153,156$ n. $99,162-166$ n. 137 , 168-169 n. 147, 171, 200, 203-206 n. 20, 212 n. 48, 220-235, 296 n. 1, 298-299, 302-306 n. 32, 308-317, 320, 324, 327 n. $129,133,328,331,333,336-340,363$

Saami $\quad 76$

Innocent passage 156 n. 101, 169 n. 146, 199, $25^{2}, 260$

International Convention for the Regulation of Whaling (ICRW) 94, 319

International Convention on Maritime Search and Rescue (SAR) $\quad 25^{8}$

International Convention on Oil Pollution Preparedness, Response and Co-operation (OPRC) 258 n. 8 o
International Convention Relating to Intervention on the High Seas in Cases of Oil Pollution Casualties $25^{8}$ n. 80 International Convention on Standards of Training, Certification and Watchkeeping for Seafarers (sTcW) $\quad 25^{\circ}$ International Covenant on Civil and Political Rights (ICCPR) 72 n. 29, 315

International financial institutions (IFIS) 51

International Labour Organization (ILO) ILO Convention No 169 73, 75, 78, 93 International Maritime Organization (IMO) International Convention for the Control and Management of Ships' Ballast Water and Sediments $\quad 261,267$ International Polar Year 14, 33 n. 106, 140, 161

International Seabed Authority 187 International Whaling Commission (IWC) 94, 299, 318-319, 323, 325-334, 337

Inuit Circumpolar Council (ICC)

Circumpolar Inuit Declaration on Sovereignty in the Arctic 136, 204 n. 13

International Standardization Organization

(ISO) $\quad 354-356,358$

Italy $45,243,244$ n. 17, 293

Japan 2 n. $8,13,22,34,45,83$ n. $62,160,172$, 184, 208 n. 30, 244 n. 17, 280, 292-293, 326

Kara Sea 173

Kayaktivists $\mathbf{0 o 0}$

LOSC, see UNCLOS

Manhattan, ss 129

Marine mammals $4,6-7,86,124,214-216$, 248, 296-299, 317, 321 n. 106, 322 n. 108, $323-325,332,337-341,363$

Marine pollution $\quad 130,180,183,255,262$ n. 93, 343

Marine protected areas (MPA) 3, 33, 139, 151, 161, 293

Maritime boundary delimitation $\quad 181,197$

Maritime security $157,182,190,262$ n. 92 
Maritime zones

Continental shelf $82,89,90,136,138,149$, $154-155,183,188,190$ n. 101, 196-197, 344 n. 11,354 n. $47,357-35^{8}$

EEZ $70,89,105,172$ n. $3,180,183^{-184}, 196$, 197 n. $147,251,254,256$ n. 74, 266 n. 104, 281, 344 n. 11

High Seas $\quad 2,7,33,90,160,183,214,251$, 258,274 n. 5 , $275^{-278}$ n. $25,280-281$, 287-288, 292, 294

Territorial sea $89,105,183,196,251-254$, 256, 344 n. 11

MARPOL $\quad 250,257,258,263,264$ n. 98 , $267-268$

Military $\quad 43,60-61,105,108$ n. 50, 120, 126 n. 19, 127-128, 134 n. 39, $135^{-136}$ n. $44,139,149-151$ n. 87,153 n. 92,174 , 175, 184-185 n. 79, 188, 190-194, 197-198, 201 n. $6,204,245$ n. 21,257 n. 76

North East Atlantic Fisheries Commission

(NEAFC) 279, 285, 292-294

North Atlantic Marine Mammal Commission (NAMMCO) 325, 337 n. 193

North Pacific Fur Seal Convention 210 n. 41

Netherlands $45,78,208,243$ n. 12, 254 n. 64, 296 n. 1, 304 n. 21, 318 n. 92

New Zealand 326 n. 123

NGO $37-38$

Nordic Cooperation 41-42, 57

Nordic Council 19 n. 48, 23 n. 68, 52 n. 46, 56,57 n. $62,60,75$ n. 36,104 n. 3 , 108 n. 50, 122, 153, 207, 212 n. 53, 277 n. 20,279 n. $34,304,311$ n. 61,314 n. 73 , 329 n. 142

North Atlantic Treaty Organization (NATO) 42, 44, 46, 48, 61, 191

North Pole 104, 106, 120, 126, 149, 160 n. 116, $276,287,345$

Northeast Passage 120 n. 5,157 n. 104

Northern Sea Route (NSR) 6, 49, 156 n. 99, 157, 180, 247

Northwest Passage $\quad 120,123,125^{-126}, 129$, $131,133,138,139$ n. 50, 155, 156, 201 n. 4, 247,253

Norway

Norwegian Intelligence Service (NIS) 188, 193
Svalbard 82 n. 59, 104-106, 108, 109, 110 n. $55,187,246$ n. $30,251,263$ n. 97 , 266 n. 104

Novaya Zemlya 247

Oil \& gas

Safety of offshore oil \& gas operations

89

Arctic Council Offshore Oil \& Gas

Guidelines 72,85

EU Directive 2013/30/EU 89

ISO 89

OSPAR $\quad 293^{-294}$

Pacific Ocean 217-218

Protection of the Arctic Marine Environment

(PAME) Working Group 144, 248, $25^{8}$ n. 77,275 n. 7,355

Paris Agreement 148

Particularly Sensitive Sea Area (PSSA) $\quad 263$

Pechora Sea 173

Persistent Organic Pollutants 16, 34 n. 111, 132

Pinniped $300 \mathrm{n} .5$

Poland 41 n. 9, 42, 44-45, 208, 224, 243 n. 12, $251 \mathrm{n} .47$

Polar bear $\quad 33,165$ n. 134, 168-169 n. 147

Polar Code $\quad 2,15,144,157,182,239$ n. 1, 257, 259-261, 263 n. 94, 266, 268

Polluter pays principle 344

Pollution

Convention on Long-range Transboundary Air Pollution 16

Stockholm Convention on Persistent Organic Pollutants 16

Port state $18,253,256,264,266,268-270$, 272 n. 126,278

Precautionary approach $\quad 152,333,343-344$

Regional Management Fisheries Organization (RFMO) 280-281, 287-288, 291

Reindeer 21, 27-28, 76, 113, 115, 143, 208 n. 32 Resources

Fisheries $\quad 2-4,6-7,13,15^{-16}, 27,29$ n. 91, 30, 32 n. 101, 34 n. 112-113, 49 n. 35, 58, 59 n. $71,69,70,94$ n. $85,87,100-102$ n. 20,105 n. 36,109 n. 50, 110, 113, 124, 152, 16o, 168 n. 142, 215-216, 229 n. 125, 
243, 274, 275, 276, 277, 278, 279-295, 302 n. 12, 316 n. 82, 320, 337, 338 n. 197 Minerals 21, 26, 65, 84 n. $64,87,173$ n. 5 , 187

Oil \& gas 72 n. $26,82-83,89,91,96$ Berger Inquiry 130

Deepwater Horizon $\quad 89,35^{\circ}-351,366$ Safety of offshore oil \& gas operations 89

Arctic Council Offshore Oil \& Gas

Guidelines 72 n. 26,85 n. 68 EU Directive 2013/30/EU 89 Iso 89

Russian Federation

Archangelsk 54, 185

Federal Security Services of the Russian Federation (FSB) 193

Kola Peninsula $47,54,185,198$

Murmansk 43 n. $15,49,54,185$, 190-191 n. 108, 195, 198

Novaya Zemlya 157 n. 104, 247

Russian Marine Doctrine $\quad$ 174, 186

Schengen 40, 101

Search \& Rescue (SAR) 191-192, 194

Seals

EC-Seal Products 166 n. 137, 308-309, 311, 314, 333

EU Directive 83/129/EC 301 n. 9, 302, 304, 306 n. 32

EU Regulation 348/81 318, 320-321, 323

EU Regulation 3786/81 318, 320 n. 99

EU Regulation 1007/2009 297 n. 3, 298, 304, 306 n. 35, 309-310, 313 n. 69, 314 n. 72,318 n. 92

EU Regulation 737/2010 298 n. 4, 304, 305 n. 27, 307, 311 n. 59

Inuit Tapiriit Kanatami and Others $v$ European Commission 309, 310 n. 51-52, 316 n. 82

Shipping $2,4-6,27,40,49,58,120,124$, $129,133,136,141-144$ n. $64,152,156$, 157-158, 180, 182, 184-186, 188-190, 196, 199, 239-241, 243, 244 n. 16, 245 n. 22, 246-248, 249 n. 40,252 n. 53, 253 n. 59, $254-273,282,351$ n. 37,359

Singapore 208 n. 30, 244 n. 17, 293

Slovakia 42
SOLAS 250, 257, 259 n. 84

South Africa $\quad 169$

South Korea 2 n. 8, 65, 172 n. 4, 244 n. 17

Spain 78, 208, 243 n. 12, 279, 283, 317 n. 86

Special Area of Conservation (SAC) 321

STCW 250, 257

Sustainable development

Sustainable Development Working Group (SDWG) 144

Svalbard

Spitsbergen 105 n. 32-33, 109 n. $5^{1}$

Spitsbergen Treaty 109

Fisheries Protection Zone (FPZ) 105, 109

Sweden $\quad 2,19-21$ n. $55,40,45^{-48}$ n. 32, 76, 84 n. 64, 99-100 n. 9, 104, 107, 118, 172 n. $3,176,181,187,195,206-208$ n. 29 , 232, 241, 292-293, 342 n. 1, 353

Syria 146

Technical Barriers to Trade Agreement (твт) 226

Territorial sea $89,105,183,196,25^{1-254}, 25^{6}$, 344 n. 11

Tourism $\quad 2-3,14,29,69,80,87,113,120,142$, 144, 246, 247 n. 33

Transboundary impacts $90,132,279,293$

Transatlantic Trade \& Investment Partnership (TTIP) $241 \mathrm{n} .8$

Ukraine $\quad 55^{-56}, 117,138,146,15^{0-151,170,}$ 191-193, 199, 224, 242

United Kingdom Continental Shelf Act (1964) 183

United Nations Declaration on the Rights of Indigenous Peoples (UNDRIP) $73-75,78,93$

United Nations Law of the Sea Convention (LOSC)

Article $2 \quad 252$ n. 54

Article 3 211, 221 n. 92, 229 n. 126, 252, 344 n. 11

Article $7 \quad 185,196$

Article $8 \quad 186$

Articles 17-26, $25^{2}$ n. $5^{1}$

Articles 41-44, 252 n. $5^{2}$

Article 76 149, 196 n. 140, 197 n. 149

Article $92 \quad 251$ 
United Nations LOSC (cont.)

Article $94 \quad 250$ n. 43, 251 n. 45

Article $208 \quad 359$

Article 211, 251 n. 48

Article $214 \quad 359$

Article $218 \quad 267-268$

Article $221 \quad 251$

Article $234 \quad 129-130$ n. 29, 180, 253, 255,

256 n. 74, 259, 265

Article $300 \quad 269$ n. 120

Annex IV, Article 4, 264 n. 98

United States of America (USA)

Endangered Species Act $\quad 213$

Marine Mammal Protection Act (MMPA) 213, 297 n. 3

Migratory Bird Treaty Act $\quad 213$

US Coast Guard (USCG) 131, 192, 266 n. 107
Visegrad 42, 44

Vulnerable Marine Ecosystem (VME) 294

Walrus 216, 300

West Nordic Council (WNC) 41-42, 56-58, 60,207 n. 27

Whales

Whaling 26, 94, 95 n. 92, 299, 303 n. 19, 316-319, 321-334, 335 n. 181, 337-340 Aboriginal subsistence whaling (ASW) 94-95, 328, 330-334, 338, 340-341, $362-363$

Aboriginal Subsistence Whaling Sub-Committee (ASWSC) $\quad 328$

World Trade Organization (WTO) 94, 115, 165 n. 131, 166-167, 221, 222 n. 96, 223, 224-226, 229-230, 233-234, 243 n. 13, 244 n. 18 